\title{
Collision-Induced Migration of Adsorbates on Solid Surfaces: An Experimental Approach ${ }^{\dagger}$
}

\author{
I. M. Danziger and M. Asscher* \\ Department of Physical Chemistry and the Farkas Center for Light Induced Processes, \\ The Hebrew University of Jerusalem, Jerusalem 91904, Israel
}

Received: November 24, 2005; In Final Form: March 19, 2006

\begin{abstract}
Collision-induced migration (CIM) is a process in which energetic gas-phase atoms or molecules at the tail of the Boltzmann distribution enhance surface migration of adsorbates upon collision. It is believed to exist and play an important role in any realistic high pressure-high-temperature heterogeneous catalytic system. Combining supersonic beam-surface collision setup with in-situ optical second harmonic generation diffraction technique from a coverage grating, we have shown, for the first time, that indeed energetic collisions (Kr seeded in $\mathrm{He}$ ) promote surface mobility of $\mathrm{CO}-\mathrm{K}$ surface complex on $\mathrm{Ru}(001)$ with a threshold total kinetic energy of $3 \mathrm{eV}$. An average migration distance/collision of more than 30 adsorption sites was estimated from the experimental data at $\mathrm{Kr}$ total energy of $3.8 \mathrm{eV}$. This long-range migration distance per collision is understood in terms of a cascade migration mechanism, where adsorbed $\mathrm{CO}$ molecules collide and push their neighbors from high to low coverage areas, in a direction dictated by the collision momentum vector. A similar mechanism has recently been suggested to explain adsorbate mobility at high coverage induced by an STM tip.
\end{abstract}

\section{Introduction}

Diffusion of adsorbates is among those primary processes on solid surfaces that govern heterogeneous catalysis via Langmuir-Hinshelwood mechanism. In addition it dictates the growth kinetics of atomic films. Many fundamental studies have been conducted over the years to determine the atomic level mechanism and thermodynamics of this surface event. ${ }^{1,2}$ Whether a single site-hopping microscopic view based on field ion microscopy $^{1}$ or more recently following STM imaging ${ }^{3,4}$ or macroscopic studies using laser-based techniques, ${ }^{5-12}$ all surface diffusion phenomena studied so far were considered driven by the substrate thermal energy.

In recent years theoretical and experimental studies have demonstrated the potential role of energetic colliders on the reactivity of adsorbates on solid surfaces. ${ }^{13-19}$ The relevance of gas-surface collisions to realistic high-pressure industrial conditions has been discussed on the basis of the tail of the Boltzmann kinetic energy distribution at high temperature and pressure that contains significant flux of energetic gas-phase colliders. Collision-induced processes were demonstrated to include desorption (CIDes) $)^{13-17}$ and reaction (CIR). ${ }^{18,19}$ Dissociative adsorption dynamics of energetic molecular colliders on metal surfaces has been at the focus of theoretical and experimental research for more than two decades. In these studies of the role of internal degrees of freedom of the collider, statistical vs quantum mechanical theoretical approaches to describe the dynamics were presented over the years. ${ }^{20-22}$ This family of collision-induced processes is outside the scope of this study.

It is obvious that surface diffusion of adsorbates, typically characterized by an energy barrier lower than that for desorption $\left(E_{\text {diff }} \approx(0.3 \pm 0.2) E_{\text {des }}\right)$, should be significantly affected by energetic gas phase colliders. Moreover, collision-induced migration (CIM) of adsorbates is expected to result in surface

\footnotetext{
† Part of the "Chava Lifshitz Memorial Issue".

* Corresponding author. E-mail: asscher@fh.huji.ac.il.
}

reactivity that may not be possible at a given temperature without energetic collisions. It is interesting therefore to note that no previous attempt has been made so far to experimentally examine this important "new" surface elementary process, despite the potential importance it may have for understanding the details of catalytic processes at industrial high pressure and high-temperature conditions. In a theoretical introduction to the subject, based on molecular dynamics simulations, the potential effect of energetic collisions on adsorbates mobility at low and at high coverage and high pressures has been demonstrated. ${ }^{19,23}$

Here we present the first experimental attempt to study CIM. We have measured the CIM of potassium atoms in the presence of $\mathrm{CO}$ on an $\mathrm{Ru}(001)$ surface under UHV conditions, using an energetic rare gas supersonic atom beam, while in-situ monitoring the surface diffusion by means of the optical second harmonic diffraction technique.

\section{Experimental Section}

The experiments reported here were conducted in an apparatus for rare gas atomic beam-surface scattering experiments under UHV conditions described elsewhere in detail. ${ }^{17}$ Briefly, a typical base pressure of $2 \times 10^{-10}$ Torr increased to $1 \times 10^{-8}$ Torr when the beam of rare gas (Ar or $\mathrm{Kr}$ ) seeded in helium was on. Preparation of the $\mathrm{Ru}(001)$ crystal followed standard sputter-annealing cycles explained in the literature. ${ }^{24,17}$ The separate elements of the experimental setup are detailed below and schematically reproduced in Figure 1.

2.1. Atomic Beam-Surface Scattering Apparatus. To deliver energetic colliders that will be able to overcome the barrier for diffusion of the adsorbed species (see details in section 2.4 below), it was necessary to seed heavy rare gas atoms in helium, so that kinetic energies above $1 \mathrm{eV}$ could be obtained. ${ }^{25}$

A supersonic beam of $\mathrm{Kr}$ seeded in He was generated in a differentially pumped section composed of three chambers, separated by a single skimmer and two collimating apertures; the details were given elsewhere. ${ }^{17}$ Briefly, a heat-shielded 


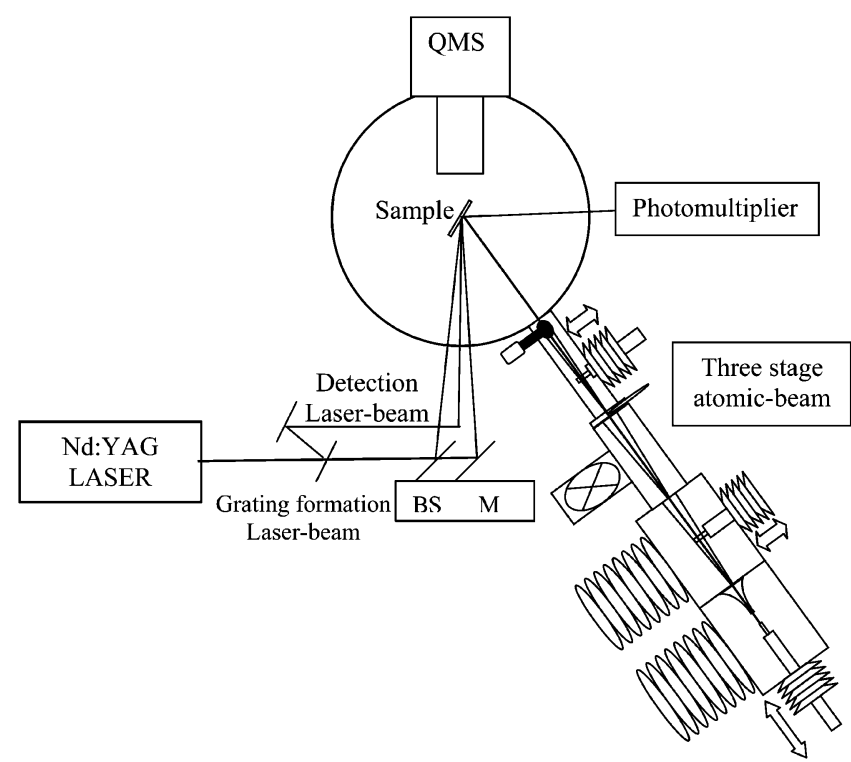

Figure 1. Schematic experimental setup combining supersonic atomic beam-surface collision ( $\mathrm{Kr}$ seeded in $\mathrm{He}$ ) with optical SHG-diffraction measurements.

ceramic nozzle with an orifice of about $60 \mu \mathrm{m}$ (Micro-Swiss) could be heated to $1500 \mathrm{~K}$ with a dc resistive heating of a 0.5 $\mathrm{mm}$ tantalum wire. A skimmer of $100 \mu \mathrm{m}$ diameter was placed about $2 \mathrm{~cm}$ away from the nozzle, and the apertures were made such that the beam diameter was slightly larger than the sample at the center of the UHV scattering chamber, a total distance of $90 \mathrm{~cm}$ away. This ensures homogeneous distribution of the rare gas atoms striking the sample. A $400 \mathrm{~Hz}$ chopper $(50 \mathrm{~mm}$ diameter, $0.5 \mathrm{~mm}$ thickness, a single $0.5 \mathrm{~mm}$ slit) stainless steel disk on a compact synchronous gyro-motor (Condor Pacific Ltd.), in the first differentially pumped chamber, has been employed to determine the velocity distributions of the incident rare gas atoms. Velocity distributions were determined at a distance of $50 \mathrm{~cm}$ from the chopper in the open ionizer of a quadrupole mass spectrometer (VG quartz) positioned perpendicular to the beam axis, inside the UHV chamber. The most probable velocity was used to define the incident kinetic energy, and from the width of the velocity distribution an energy spread of $\Delta E / E=0.4$ was deduced for room-temperature nozzle. At a nozzle temperature of $1500 \mathrm{~K}$ the distribution increased to $\Delta E / E$ $=0.5$. The gas mixture was prepared in a high-pressure (up to 6 bar) cylinder with a magnetic stirrer that generates a stable mixed gas within $30 \mathrm{~min}$. The beam cleanliness and gas mixture content were determined by quadrupole mass spectrometer (SRS) across the UHV chamber. This QMS also enabled accurate positioning of the sample in the beam path.

The absolute atomic beam flux was determined from the partial pressure rise produced by the $\mathrm{Kr}$ beam in the UHV chamber. ${ }^{17} \mathrm{We}$ measured a flux of $(1.5 \pm 0.5) \times 10^{14} \mathrm{Kr}$ atoms/ $\left(\mathrm{cm}^{2} \mathrm{~s}\right)$. Angle of incidence of the beam was fixed at $22 \pm 2$ deg with respect to the surface normal.

2.2. Optical SHG Diffraction. To follow the CIM one has to prepare coverage grating out of the potassium overlayer prior to $\mathrm{CO}$ coadsorption. The optical setup included a $p$-polarized pulsed Nd:YAG laser (Quantel YG585) at its fundamental wavelength of $1064 \mathrm{~nm}$, repetition rate of $10 \mathrm{~Hz}$, and pulse duration of $10 \mathrm{~ns}$. The same laser produced both the coverage grating via laser-induced thermal desorption (LITD) and the probe beam using a small fraction of the fundamental laser beam. The probing method was an optical second harmonic $(\mathrm{SH})$ diffraction from the adsorbate grating. In these experiments the split ratio ( $r$ in eq 1, section 3.2) for the heating (LITD) laser beam was 0.5 ; i.e., the laser was split into two beams of approximately equal intensity. The two beams strike the potassium-covered $\mathrm{Ru}(001)$ surface at incident angles of $\phi=$ $\pm 5^{\circ}$ from the normal to the surface. These beams spatially overlapped at the center of the sample having a spot size of 0.4 $\mathrm{cm}^{2}$. The maximum total effective laser power density of the high-power fraction of the LITD laser actually absorbed by the metal/pulse (both beams, calculated as $1-R, R$ being the reflectivity at $1064 \mathrm{~nm}$ of about 0.75$)$ was $12 \mathrm{MW} / \mathrm{cm}^{2}(150$ $\mathrm{mJ} /$ pulse). Repeated exposure to the upper limit of the desorption laser power did cause some damage to the crystal, as evidenced by a change in the measured diffusion rate. Annealing at 1600 $\mathrm{K}$ for more than $10 \mathrm{~min}$ restores the original structure almost completely. A spatial intensity modulation is formed under these conditions due to interference between the two beams. As a result, a periodic potassium coverage modulation is created in a gratinglike form. ${ }^{11}$

The small fraction of about $4 \%$ reflected probe beam at 1064 $\mathrm{nm}$ hits the center of the sample at an incident angle of $43^{\circ}$ from the normal to the surface having a spot size of $0.05 \mathrm{~cm}^{2}$ to ensure that the probe laser falls within the area of grating formation. The fundamental laser intensity was at most $3.5 \mathrm{MW} /$ $\mathrm{cm}^{2}$, which corresponds to a maximum change in surface temperature of $80 \mathrm{~K}$ (above the crystal temperature of $95 \mathrm{~K}$ ) for the duration of the laser pulse. This ensures that no diffusion of potassium due to probe laser heating takes place, as verified experimentally elsewhere. ${ }^{11}$

\section{Results and Discussion}

The various aspects of the unique setup for measuring collision-induced migration (CIM) are described below, pertaining to $\mathrm{CIM}$ of a supersonic beam of $\mathrm{Kr}$ with the modulated $\mathrm{CO}-\mathrm{K}-\mathrm{Ru}(001)$ system.

3.1. Potassium on $\mathbf{R u}(\mathbf{0 0 1})$. Preparation of well-calibrated potassium surface coverage relies on a detailed knowledge of the coverage-dependent desorption kinetics. ${ }^{6,12,26,27}$ Assuming a preexponential factor for desorption of $10^{13} \mathrm{~s}^{-1}$, the activation energy for desorption of potassium from $\mathrm{Ru}(001)$ was determined (line shape analysis) to decrease from 65 to $25 \mathrm{kcal} / \mathrm{mol}$ as coverage increases from near zero up to a monolayer. ${ }^{12,27}$ Reproducible coverage could be obtained by dosing potassium to more than a monolayer, in-situ monitored by optical second harmonic generation (SHG), and then heating the surface to a given temperature at a heating rate of $10 \mathrm{~K} / \mathrm{s}$. Heating to $335 \mathrm{~K}$ produces one monolayer of potassium on the surface. A full monolayer (ML) of potassium is defined as that just after the disappearance of the multilayer TPD peak at $335 \mathrm{~K}$, where SHG signal intensity is just over its maximum. ${ }^{27}$

3.2. Grating Formation and SHG Measurement. The optical second harmonic ( $\mathrm{SH}$ ) response of a solid surface is typically sensitive to changes in adsorbate-surface electronic structure via the second-order susceptibility of the substrate. On a clean substrate the strong SH response of the potassiumcovered surface is due to the particularly large second-order susceptibility of the alkali-metal complex at the fundamental laser wavelength of $1.064 \mu \mathrm{m} .^{28,29}$

Under the conditions described in section 2.2, a spatial intensity modulation is formed due to interference between the two beams on the surface. The laser power modulation along the surface ( $x$-axis) is given by ${ }^{7-9}$

$$
I(x)=I_{0}+2 I_{0} \sqrt{r(r-1)} \cos (2 \pi x / \omega)
$$




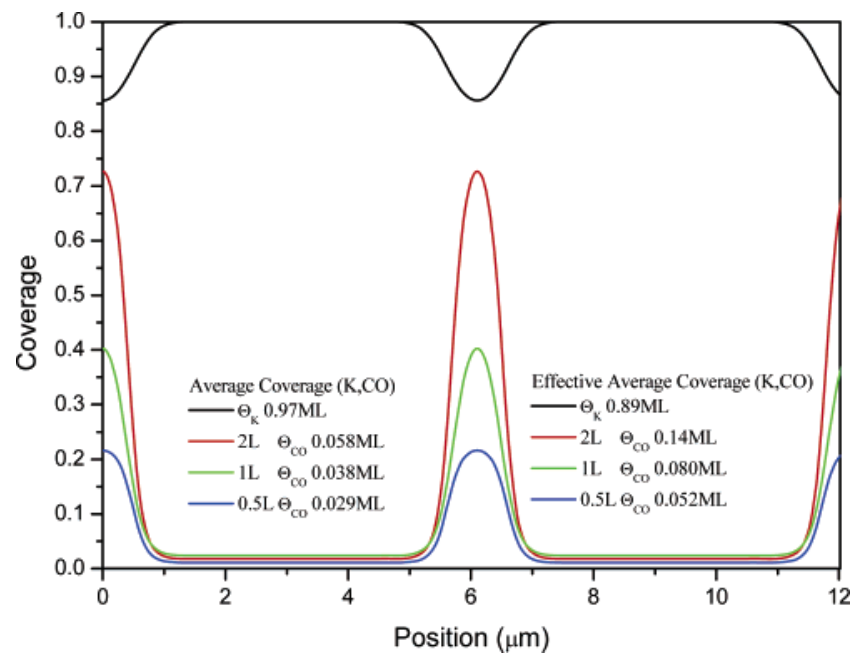

Figure 2. Calculated shallow potassium coverage grating (upper curve) with corresponding $\mathrm{CO}$ coverages obtained following exposure to the indicated $\mathrm{CO}$ exposures in langmuirs $\left(1 \mathrm{~L}=10^{-6}\right.$ Torr s). For definitions of average coverage and effective average coverage, see text and ref 28 .

where $I_{0}$ is the incident laser intensity, $r$ is the intensity fraction determined by the beam splitter, $\omega$ is the grating period as given by the Bragg equation, and $x$ is the distance parameter along the surface. This periodic intensity modulation leads to the formation of a potassium coverage grating, due to partial potassium desorption at the hot, high intensity (constructive interference) troughs. This is given by

$$
\theta(x)=\theta_{0}+\sum_{n=1}^{\infty} \Theta_{n} \cos (2 \pi n x / \omega)
$$

At initial potassium coverage of $1 \mathrm{ML}$, an LITD absorbed laser power density of $12 \pm 3 \mathrm{MW} / \mathrm{cm}^{2}$ was employed to create a potassium grating. This laser power ensures that the potassium coverage modulation is relatively shallow and the surface is not damaged. As a result, the effective potassium coverage at the bottom of the troughs was 0.90 to $0.85 \mathrm{ML}$ and the grating period, given by $d=\lambda / 2 \sin \phi$, was $6 \mu \mathrm{m}\left(\phi= \pm 5^{\circ}\right){ }^{11,12}$ see Figure 2.

The diffracted SH signal was optically filtered and dispersed via a $60^{\circ}$ optical prism to get the optimum separation between 1064 and $532 \mathrm{~nm}$ and best signal-to-noise ratio at the detection wavelength of $532 \mathrm{~nm}$.

The intensity of the diffracted order $n$ is given by

$$
S_{n}(t)=S_{n}(0) \exp \left[-8 n^{2} \pi^{2} D\left(\Theta_{0}\right) t / \omega^{2}\right]
$$

where $n$ is the diffraction order ( \pm 1 in this experiment), $\omega$ is the grating period, and $D\left(\Theta_{0}\right)$ is the diffusion coefficient, which is assumed to be constant along the $x$-axis due to the shallow coverage modulation. ${ }^{11}$ Analysis of the exponential decay of the first-order diffraction peak at several different temperatures provides $D\left(\Theta_{0}, T\right)$. This can be transformed into an average displacement distance as will be discussed below.

3.3. $\mathbf{C O}-\mathbf{K}-\mathbf{R u}(\mathbf{0 0 1})$. Clean potassium has turned out to be too sensitive grating to be exposed to the energetic atomic beam for the relatively long duration of the CIM experiment. Background gas impurities were sufficient to modify the SHG signal and the thermal diffusion kinetics of clean potassium, as indicated from our preliminary study. As a result, we had to modify the surface by exposing it to $\mathrm{CO}$. It was previously shown on $\operatorname{Re}(001)^{30}$ and $\operatorname{Ru}(001)^{31}$ that $\mathrm{CO}$ sticks preferentially and with higher probability onto the areas inside the grating troughs with lower potassium coverage. As a result of very small amounts of coadsorbed $\mathrm{CO}$, the barrier for surface diffusion of potassium has been dramatically affected. In the case of $\mathrm{Ru}(001)$ we measured an increase of the activation energy for potassium diffusion from $2.3 \mathrm{kcal} / \mathrm{mol}$ for pure $\mathrm{K}$ up to $17 \mathrm{kcal} / \mathrm{mol}$ when $\mathrm{CO}$ at average coverage of $0.14 \mathrm{ML}$ was coadsorbed. The high barrier for diffusion typical to the coadsorption of $\mathrm{CO}$ significantly stabilizes the system against impurities. In addition, it prevents any thermal effect on the diffusing atoms by the probe SHG laser that heats the surface by about $80 \mathrm{deg}$ (for the duration of the laser pulse, about $10 \mathrm{~ns}$ ). The first-order diffracted SH signal was stable under such coadsorbed system to within $10 \%$ during the entire diffusion measurement time while exposed to background gas and the continuous SHG probe laser.

Finally, the coverage profiles of $\mathrm{CO}$ on the modulated potassium density were simulated on the basis of standard LITD equations. ${ }^{31-35}$ These are shown for demonstration in Figure 2 for initial potassium coverage of $1 \mathrm{ML}$ (before grating formation) and several $\mathrm{CO}$ exposures. The final $\mathrm{CO}$ coverage was obtained from separate $\mathrm{CO}$ sticking measurements on top of various potassium coverages for calibration purposes (these are not shown here). Note that the actual CO coverage within the lower coverage potassium troughs is significantly higher than the average coverage indicated in the figure. Details were given elsewhere. ${ }^{30,31}$

3.4. Collision-Induced Migration: $\mathrm{Kr}$ Striking $\mathrm{CO}-\mathrm{K}-$ $\mathbf{R u}(001)$ Coverage Grating. 3.4.1. Migration Distance/Collision, $\Delta r$. Once the periodic coverage grating of $\mathrm{CO}$ on the potassium grating template has been prepared, it was exposed to the supersonic $\mathrm{Kr}$ beam. This was done while the optical second harmonic first-order diffraction signal has continuously been monitored in real time. In such a delicate experiment it is crucial to make sure that the decay of the first-order diffracted peak arises only from CIM and that it is not affected by background gas. To be able to subtract the background contribution to the decaying signal, the first-order signal was recorder in the following way: once with only background gases, then with a pure He beam striking the sample (not shown), and finally with the actual supersonic Kr beam on, but blocked by a flag, thus preventing beam collision with the surface for the entire time duration of a typical decay period. The last background curve is then subtracted from that recorded with the $\mathrm{Kr}$ beam striking the surface. In Figure 3, decay curves of the first-order $\mathrm{SH}$-diffracted signals are shown at two $\mathrm{Kr}$ seeding conditions of $8 \%$ (Figure $3 \mathrm{~A}$ ) and 2\% (Figure 3B) in He. These include background effect, flagged beam (shown only for the case of $8 \%$ seeded beam in Figure 3A), and the actual effect of $\mathrm{Kr}$ beam striking the sample at different kinetic energies. In Figure 4, the decay curves obtained after subtraction of background effects are shown for three $\mathrm{Kr}$ kinetic energies. It is evident that the decay rate, which reflects smearing out the one-dimensional grating and therefore the collision-induced migration process of the adsorbates, is indeed enhanced by the energetic collider.

Simulations of the level of smearing reflected by the decay of the first-order diffracted peak are demonstrated in Figure 5. The coverage profiles of potassium were calculated using the known laser intensity/surface temperature profiles and therefore the extent of local desorption along the distance parameter- $x$. Similar curves were shown before and are demonstrated in detail in refs 8, 9-11, 31, and 32. Points $\mathrm{A}-\mathrm{E}$ in Figure 5 (lower frame) were picked up arbitrarily in time simulating the continuous collision event while the SH-diffracted first-order 



Figure 3. Decay of first-order SHG-diffracted signal during CIM as a result of $8 \% \mathrm{Kr}$ seeded in $\mathrm{He}(\mathrm{A})$ and $2 \% \mathrm{Kr}$ in $\mathrm{He}(\mathrm{B})$ at the indicated $\mathrm{Kr}$ kinetic energies obtained by heating the ceramic nozzle (Tn). The background UHV and flagged beam signals are explained in the text. The sample temperature was $95 \mathrm{~K}$.



Figure 4. Decay of the first-order SHG diffracted signal obtained at the three indicated $\mathrm{Kr}$ kinetic energies following background subtraction of the signals shown in Figure 3. The exponential decay constants $\mathrm{K}$ are obtained from the best fit to the subtracted signals.

peak intensity decays. These points are correlated with corresponding profiles of the coverage grating as they evolve during a thermal diffusion process. This is used as a model to visually follow the CIM process (Figure 5, top frame).

The main question arising from the results displayed in Figures 3 and 4 and the simulation of Figure 5 is to what extent the data can be translated into a single parameter, $\Delta r$, that should reflect the (one-dimensional) migration distance of an adsorbate/ collision with a $\mathrm{Kr}$ atom. Typically, an average hopping distance such as $\langle\Delta r\rangle$ is correlated with the diffusion coefficient $D$ $\left(\mathrm{cm}^{2} / \mathrm{s}\right)$, in a thermal diffusion, via the Einstein expression, $D$ $=<(\Delta r)^{2}>/ 4 t$, where $t$ is the diffusion time. ${ }^{1}$

Examination of the CIM process, in contrast, requires the evaluation of an average migration distance/collision once the total migration length is known and so is the flux of incident colliders. On the basis of the example given in Figure 5, it is possible to correlate the decay curve in Figure 4, e.g. at $\mathrm{Kr}$ energy of $3.8 \mathrm{eV}$, with the curve in Figure 5, point $\mathrm{C}$ after about $500 \mathrm{~s}$ of the real experiment; see Figure 4. At this point, most of the $\mathrm{CO}-\mathrm{K}$ population within the filled regions of the coverage-grating troughs have migrated to the regions with smaller $\mathrm{CO}$ population and higher potassium coverage, as a result of collisions. This trend is also expected during onedimensional thermal diffusion as dictated by local chemical potential gradient. To fill these troughs the $\mathrm{CO}-\mathrm{K}$ species need to migrate over a distance of about $1 \mu \mathrm{m}$ on average, half the width of the potassium coverage troughs. This means that during a period of $500 \mathrm{~s}$ (point $\mathrm{C}$ in Figure 5), about 100 collisions/ $\mathrm{CO}-\mathrm{K}$ complex take place, considering the incoming flux of $10^{14} \mathrm{Kr}$ atoms $\mathrm{cm}^{-2} \mathrm{~s}^{-1}$.

It means that, at a total $\mathrm{Kr}$ kinetic energy of $3.8 \mathrm{eV}$, on average an adsorbate migrates a distance of $1 \mu \mathrm{m}$ or $\langle\Delta r\rangle=$ 

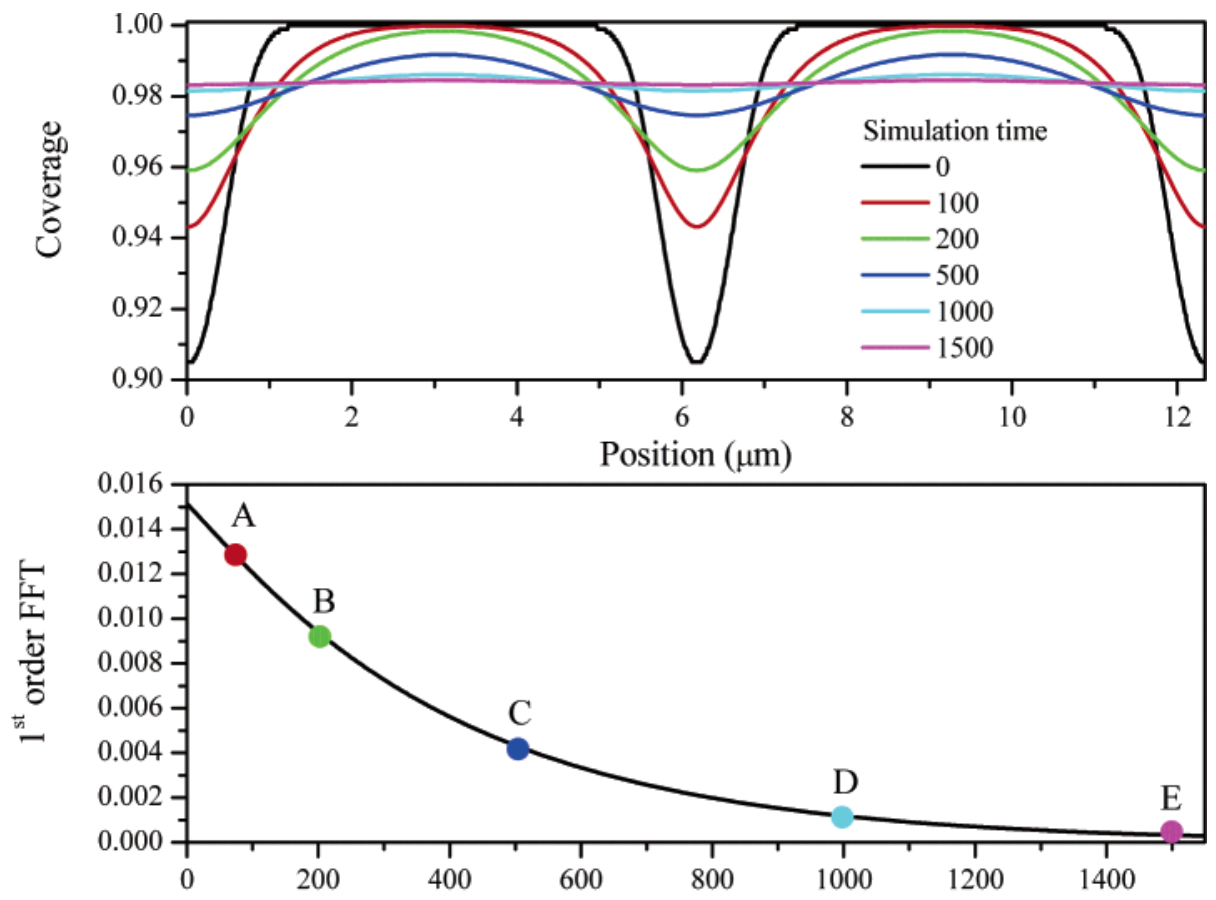

Simulation time

Figure 5. Simulated potassium coverage grating smearing out (top frame) and the corresponding exponential decay of the first-order Fourier component squared, reflecting the modulated coverage becoming homogeneous. Points $\mathrm{A}-\mathrm{E}$ on the decay curve (bottom frame) were chosen arbitrarily to demonstrate the development in time of the coverage profile.



Figure 6. Average migration distance, $\Delta r$, derived from Einstein diffusion expression, as a function of $\mathrm{Kr}$ collision energy. (It is assumed that only $30 \%$ of the total kinetic energy is actually transferred to the adsorbate, the rest to the substrate.)

$100 \pm 20 \AA$ A/collision, namely hopping over $20-30$ potassium adsorption sites (assuming $(\sqrt{ } 3 \times \sqrt{ } 3) R 30^{\circ}$ overlayer structure of $\mathrm{K}$ ) at this collision energy. We argue that the data points are consistent with migration of $\mathrm{CO}$ molecules without their potassium "counteratom", in a cascade mechanism and not the diffusion of the entire complex. This is discussed below in more detail.

As shown in Figure 6, the dependence of $\langle\Delta r\rangle$ on incident energy has a threshold: the onset for effective CIM appears to be near collision energy of $1.0 \pm 0.15 \mathrm{eV}$ (total energy of 3.3 $\mathrm{eV}$, assuming energy transfer directly to the adsorbates is near $30 \%$ (upper limit) of the total incident colliders' energy-the rest goes to the reflected collider (about 25\%) and the substrate $\left.{ }^{19}\right)$.
3.4.2. CIM Derived from the Diffusion Coefficient D $\left(\mathrm{cm}^{2} / \mathrm{s}\right)$. An alternative way to estimate the migration distance is based on the diffusion coefficient ("migration rate") directly derived from the initial exponential decay of the first-order $\mathrm{SH}$ diffracted peak, as done in the case of thermal diffusion. ${ }^{8-12}$

A major assumption here is that the basic thermodynamic rules that govern one-dimensional thermal diffusion driven by local chemical potential gradient lead to similar results when the driving force is collisions rather than thermal motion.

Once a migration rate $D$ is extracted this way, the migration distance/collision $\Delta r$ can be obtained using the Einstein equation $\left(D\left(\mathrm{~cm}^{2} / \mathrm{s}\right)=<(\Delta r)^{2}>/ 4 t\right)$, where the time parameter $(t)$ is the interval between collisions/site, about $1 \mathrm{~s}$ in our experimental conditions. The results obtained this way, shown in Figure 7, are similar to within a factor of 2 with the analysis discussed in section 3.4.1 and displayed in Figure 6. Moreover, it is interesting to note that the migration rate $D$ obtained via CIM at surface temperature of $95 \mathrm{~K}$, of the order of $10^{-12 \pm 2} \mathrm{~cm}^{2} / \mathrm{s}$, is almost identical with the rates of thermal diffusion obtained for this system at $280 \mathrm{~K} .{ }^{31}$

3.5. CIM Mechanism. Collision-induced migration model calculations have been performed earlier in our group by employing molecular dynamics (MD) simulations to describe CIM of nitrogen molecules adsorbed on clean $\mathrm{Ru}(001){ }^{19,22}$ These model calculations have suggested that the migration distance of an adsorbate as a result of energetic collisions becomes significantly shorter as the adsorbates coverage increases. Since in our current system the adsorbates coverage is close to unity, we would expect rather short average migration distance even at high collision energies.

One way to explain the relatively long hopping distance/ collision obtained from the analysis of the data in Figures 3 and 4 and shown in Figures 6 and 7 is the following: Assume the energetic collisions lead to the migration of $\mathrm{CO}-\mathrm{K}$ complex molecules but in addition and with higher probability to migration of detached $\mathrm{CO}$ molecules from low potassium 


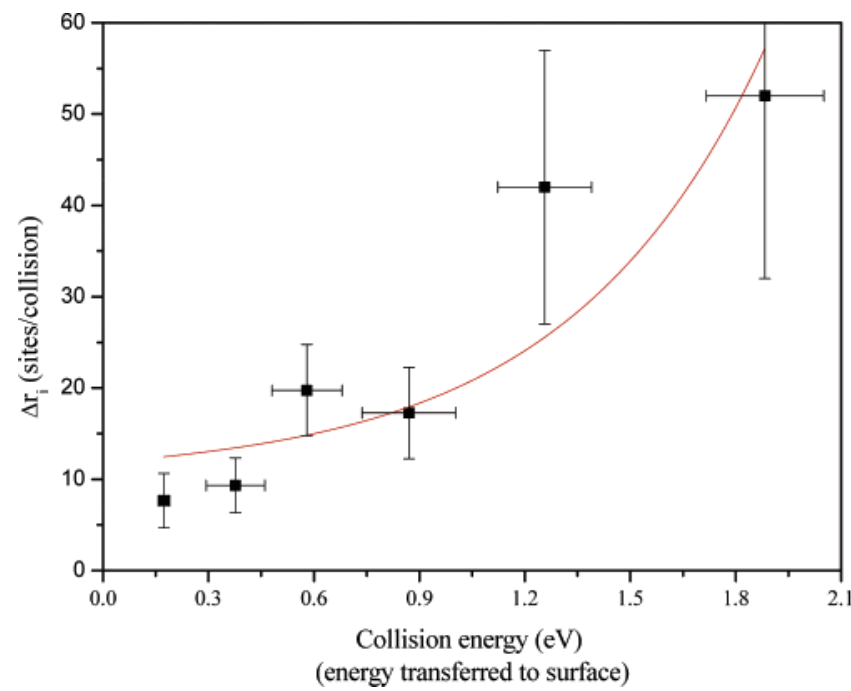

Figure 7. Average migration distance, $\Delta r$, derived from the decay constant of the first-order diffracted signal, as a function of $\mathrm{Kr}$ collision energy.

\section{Collision induced migration (CIM)}

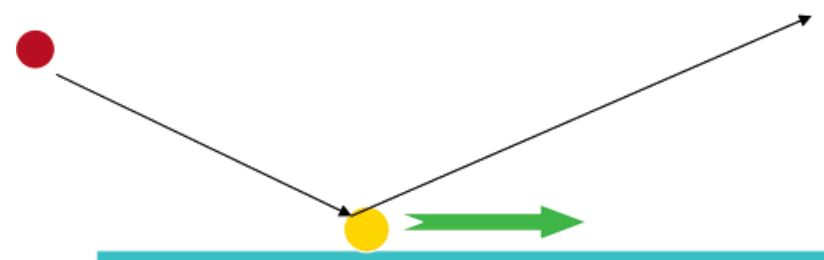

Low Coverage - Sliding

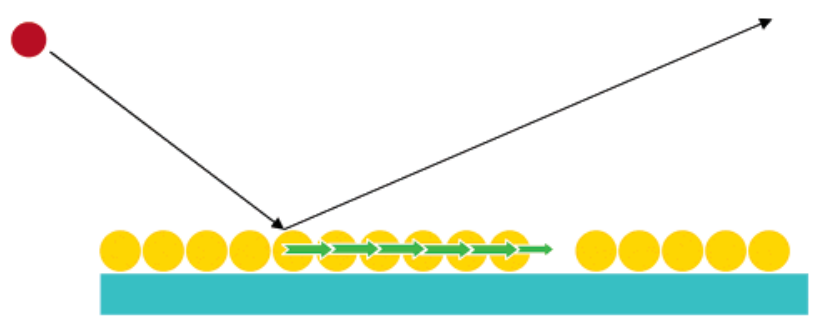

High Coverage - Cascade migration

Figure 8. Cascade mechanism for collision-induced migration at high coverage.

coverage sites within the troughs to higher potassium coverage sites where there is lower coverage of CO; see Figure 2. This is more probable since the $\mathrm{CO}$ on top of potassium is at rather low coverage. From the point of view of the observed $\mathrm{SH}$ diffracted signal, such CIM mechanism would result in effective smearing of the modulated $\mathrm{CO}-\mathrm{K}$ complex coverage in the same way, since quenching of the SHG signal by $\mathrm{CO}$ is so effective. $^{31}$

This can be considered a cascade motion of the CO molecules triggered by the energetic collisions. The result of collisions of this kind is an effective advance of empty sites that act like "holes" on the opposite direction, namely from the troughs toward the high potassium coverage. This way the original potassium density gradient effectively vanishes. A schematic view of the cascade mechanism is shown in Figure 8 . The cascade idea has previously been suggested and was demon- strated for the first time in the $\mathrm{CO} / \mathrm{Cu}(111)$ system employing STM manipulations at $4 \mathrm{~K}$ imaging. ${ }^{36}$ Similar tip-induced manipulation was more recently shown also in the case of benzene molecules adsorbed on the smooth $\mathrm{Au}(111)$ also at 4 $\mathrm{K} .{ }^{37}$ In this study the authors have analyzed the data concluding that up to 10-12 molecules are involved in a single tip-induced perturbation-cascade. Considering the strong "perturbation" induced by the energetic $\mathrm{Kr}$ collider at more than $1 \mathrm{eV}$ direct energy transfer to an adsorbate, compared with the gentle STM tip effect, one may rationalize the 3-5 times longer cascade distances observed in the CIM experiments discussed in this work.

\section{Conclusions}

We have developed a setup that experimentally demonstrates, for the first time, the concept of collision-induced migration on surfaces. Combining a supersonic seeded rare gas atomic beam-surface collision setup with in-situ optical second harmonic generation diffraction technique from a coverage grating, we have shown that indeed energetic collisions promote surface mobility with a threshold total kinetic energy of $3 \mathrm{eV}$. Seeded Krypton in helium has been used to induce surface mobility of a $\mathrm{CO}-\mathrm{K}$ surface complex on top of a wellcharacterized $\mathrm{Ru}(001)$ under UHV conditions. Migration distance/ collision has been derived from the experimental data with more than 30 adsorption sites as the average migration distance at $\mathrm{Kr}$ total energy of $3.8 \mathrm{eV}$. The migration rates measured via CIM at surface temperature of $95 \mathrm{~K}$ and collider energy of 3.8 $\mathrm{eV}$ are practically identical with thermal diffusion rates of the $\mathrm{CO}-\mathrm{K}$ system on $\mathrm{Ru}(001)$ at $280 \mathrm{~K}$. This surprisingly long migration distance/collision is understood as a demonstration for a cascade migration mechanism, where, following energetic collisions with gas-phase species, $\mathrm{CO}$ molecules collide and push their neighbors from high to low coverage areas, in a direction dictated by the collision momentum vector.

Acknowledgment. This research was partially supported by a grant from the U.S.--Israel Binational Science Foundation and by the Israel Science Foundation. The Farkas center is supported by the Bundesministerium fur Forschung und Technologie and the Minerva Gesellschaft fur die Forschung mbh.

\section{References and Notes}

(1) Ehrlich, G.; Stolt, K. Annu. Rev. Phys. Chem. 1980, 31, 603.

(2) Gomer, R. Rep. Prog. Phys. 1990, 53, 917.

(3) Zambelli, T.; Trost, J.; Wintterlin, J.; Ertl, G. Phys. Rev. Lett. 1996, 76, 795. Wintterlin, J.; Trost, J.; Renisch, S.; Schuster, R.; Zambelli, T.; Ertl, G. Surf. Sci. 1997, 394, 159.

(4) Mitsui, T.; Rose, M. K.; Fomin, E.; Ogletree, D. F.; Salmeron, M. Science 2002, 297, 1850 .

(5) Mak, C. H.; Koehler, B. G.; Brand, J. L.; George, S. M. J. Chem. Phys. 1987, 87, 2340.

(6) Westre, D.; Brown, D. E.; Kutzner, J.; George, S. M. J. Chem. Phys. 1996, 104, 7313. 2883

(7) Zhu, X. D.; Th. Rasing, Shen, Y. R. Phys. Rev. Lett. 1988, 61,

(8) Reider, G. A.; Hoefer, U.; Heinz, T. F. Phys. Rev. Lett. 1991, 66, 1994

(9) Zhu, X. D.; Lee, A.; Wong, A.; Linke, U. Phys. Rev. Lett. 1992, $68,1862$.

(10) Rosenzweig, Z.; Farbman, I.; Asscher, M. J. Chem. Phys. 1993, 98, 8277.

(11) Zhao, W.; Verhoef, R. W.; Asscher, M. J. Chem. Phys. 1997, 107, 5554

(12) Kerner, G.; Danziger, I. M.; Zhao, W.; Asscher, M. NATO Advanced Research Workshop on "Collective Surface Diffusion Coefficients Under Non-Equilibrium Conditions"; Tringides, M., Chevoj, Z., Eds.; Kluwer Academic Publishers: Dordrecht, The Netherlands, 2000. 2408 . 
(14) Zeiri, Y. Surf. Sci. 1990, 231, 408.

(15) Beckerle, J. D.; Johnson, A. D.; Ceyer, S. T. J. Chem. Phys. 1990, 93 (6), 4047.

(16) Szulczewski, G.; Levis, R. J. J. Chem. Phys. 1994, 101 (12), 11070

(17) Romm, L.; Zeiri, Y.; Asscher, M. J. Chem. Phys. 1999, 110 (6), 3153.

(18) Åkerlund, C.; Zoric, I.; Kasemo, B. J. Chem. Phys. 1998, 109, 737.

(19) Asscher, M.; Zeiri, Y. J. Phys. Chem. 2003, 107 (29), 6903-19.

(20) Weaver, J. F.; Carlsson, A. F.; Madix, R. J. Surf. Sci. Rep. 2003, $50,107$.

(21) (a) Ukraintsev, V. A.; Harrison, I. J. Chem. Phys. 1994, 101 (2), 1564. (b) Bukoski, A.; Abbott, H. L.; Harrison, I. J. Chem. Phys. 2005, $123,094707$.

(22) Harris, J.; Luntz, A. C. Surf. Sci. 1993, 287/288, 56.

(23) Romm, L.; Zeiri, Y.; Asscher, M. J. Chem. Phys. 1999, 110 (22), 11023

(24) Zeiri, Y. J. Chem. Phys. 2000, 113, 3868.

(25) Pfnur, H.; Menzel, D. 1983, 79 (5), 2400.

(26) Miller, D. R. In Atomic and Molecular Beam Methods; Scoles, G., Ed.; Oxford University Press: New York, 1992; Vol. 1, Chapter 2, p 14
(27) Bonzel, H. P.; Pirug, G. In The Chemical Physics of Solid Surfaces: Coadsorption, Promoters and Poisons; King, D. A., Woodruff, D. P., Eds.; Elsevier Science: Amsterdam, 1993; Vol. 6.

(28) Verhoef, R. W.; Zhao, W.; Asscher, M. J. Chem. Phys. 1997, 106 (22), 9353.

(29) Liebsch, A. In Laser spectroscopy and photochemistry on metal surfaces; Dai, H.-L.; Ho, W., Eds.; Advanced Series in Physical Chemistry

Vol. 5; World Scientific Publishing: Singapore, 1995; Part I, p 54.

(30) Tom, H. W. K. Mate, C. M.; Zhu, X. D.; Crowell, J. E.; Shen, Y. R.; Somorjai, G. A. Surf. Sci. 1986, 172, 466.

(31) Zhao, W.; Asscher, M. Surf. Sci. 1999, 429, 1.

(32) Danziger, I. M.; Hallac, B.; Asscher, M. J. Phys. Chem. B 2004, $108,17851-56$

(33) Burgess, D., Jr.; Stair, P. C.; Weitz, E. J. Vac. Sci. Technol., A 1986, 4, 1362.

(34) Rosenzweig, Z.; Asscher, M. J. Chem. Phys. 1992, 96 (5), 4040

(35) Danziger, I. M.; Asscher, M. Manuscript in preparation.

(36) Heinrich, A. J.; Lutz, C. P.; Gupta, J. A.; Eigler, D. M. Science 2002, 298, 1381

(37) Han, Mantooth, B. A.; Charles, E.; Sykes, H.; Donhauser, Zachary J.; Weiss, Paul S. J. Am. Chem. Soc. 2004, 126, 10787. 